\title{
Freezing of Reality: Is Flow of Time Real?
}

\author{
Bernard Korzeniewski \\ Faculty of Biochemistry, Biophysics and Biotechnology, Jagiellonian University, Krakow, Poland \\ Email: bernard.korzeniewski@gmail.com
}

Received 23 May 2016; accepted 30 July 2016; published 3 August 2016

Copyright (C) 2016 by author and Scientific Research Publishing Inc.

This work is licensed under the Creative Commons Attribution International License (CC BY). http://creativecommons.org/licenses/by/4.0/

(c) (i) Open Access

\begin{abstract}
In physics, space-time is frequently treated as a congealed timeless block, in which there is no place for the unidirectional and irreversible flow (arrow) of time and the present. However, we receive the world subjectively as "happening" in time, which is inseparably associated with the psychical feeling of the present, separating the past and the future. This article postulates that these phenomena exist in a completely real manner. An irreversible process called here the "freezing of reality" is responsible for this. It is directly associated with the collapse or decoherence of the wave-function in the result of its interaction with the surroundings. These processes do not require any "measurement" or the presence of a subjective, conscious observer.
\end{abstract}

\section{Keywords}

Quantum Mechanics, Collapse of Wave-Function, Thermodynamic Arrow of Time, Biological Arrow of Time, Psychical Arrow of Time, The Present, Past, Future

\section{Introduction}

One of the key physical-philosophical problems concerning our universe is the matter of the flow of time, arrow of time and the concept of the present. The real flow of time, arrow (irreversibility) of time and the present do not appear in dynamic physical theories: Newton's mechanics, Maxwell's electromagnetism, Einstein's special and general theory of relativity and quantum mechanics (Weinberg, 1977; Hawking, 1988; Penrose, 2002; Coveney \& Highfield, 1991; Davies, 1996; Smolin, 2014). The only exception seems to be the discussed below collapse and decoherence of the wave-function that appear in quantum mechanics and are assumed to be irreversible phenomena. However, they are, in a sense, added ad hoc to the "core" of quantum mechanics. Generally, in dynamic theories space-time can be in principle treated as a congealed timeless four-dimensional block in which time simply constitutes one of its dimensions, and in which the entire past and future is recorded (consolidated) (see below) (Hawking, 1988; Davies, 1996; Penrose, 2002; Greene, 2005; Smolin, 2014).

The significance and novelty of this work consists in postulating that the flow of time is a completely real and objective phenomenon and in proposing its "mechanism". This mechanism is what I call "freezing of reality", 
which is equivalent to the transition of the undetermined, uncertain future into congealed, already "done" and frozen past. This transition occurs on the quantum level and is related to the collapse and decoherence of the wave function. The present is equivalent to the transition point that moves along the time axis. Therefore, the entire space-time cannot be described as a congealed timeless four-dimensional block.

Section 2 of this article describes the conception of space-time as a congealed timeless block. Section 3 discusses the thermodynamic flow of time and its relation to the increase of entropy. Section 4 is the "main" section that postulates that the flow of time (as well as past, present and future) is completely real and objective, occurs principally on the quantum level and is related to the collapse and decoherence of the wave function. Section 5 deals with the relation between the quantum and thermodynamic flow of time. Section 6 discusses the biological flow of time related to the phenomenon of life and the living individual. Section 7 deals with the psychical flow of time related to the phenomenon of self-consciousness. Section 8 is the summary.

\section{Space-Time as Congealed Timeless Block}

According to some hypotheses, there is no sense talking about time before the beginning of the universe. In other words, time has no "beginning": it emerges in a certain region of space-time (close to that what we call the Big Bang) from one of four dimensions that have a spatial character (Hawking, 1988; Davies, 1996). Due to relational conceptions, for instance Leibnitz's philosophy or Einstein's general theory of relativity, in opposition to Newton's mechanics, time does not exist in an absolute way, but only in relation to other aspects of the universe, such as curved space, matter, energy, relative speed, gravity etc., and vice versa (Davies, 1996; Greene, 2005; Smolin, 2014). However, time can still be presented in the convention of one dimension of the congealed timeless block of space-time. Smolin (2014) argues that the reality of time is ensured by ceaseless originating of new universes in the moment of the formation of black holes. In every such universe the laws of nature and values of physical constants are fixed de novo on the basis of chance or, strictly speaking, through "mutations" of laws and values in the "parental" universe (Smolin, 1997, 2014). However, this reasoning is incorrect. There is no obstacle to present every particular universe as a congealed block. This also concerns the whole sequence of maternal universes and numerous descendant universes. The only exception seems to be the moment of "choices" of laws and physical constants, provided that these choices are not determined. However, there is still no place here for the present and unidirectional real flow of time, as the mentioned "choices" are one-time events. Additionally, such "choices" occur ceaselessly in quantum systems within particular universes.

According to the theory of relativity (both special and general), the rate of the flow of time in a given system is relative and depends on the movement of the system in relation to the observer (reference frame) (and also on the intensity of the gravitational field that curves space-time). Time flows are slower on quickly moving objects (and on objects located within a strong gravitational field). The same concerns the length and mass: quickly moving objects undergo shortening and their mass increases. According to the theory of relativity, no distinguished, "rest" observer (reference frame) exists. Every movement is relative and all observers have equal rights. Does this actually take place in the scale of the whole universe? We have reasons to believe that it does not, and that a distinguished, rest reference frame that "experiences" a universal, cosmic flow of time exists. In relation to such a frame, the sum of kinetic energy ${ }^{1}$ in sufficiently large scales of magnitude (distance) is minimum, while their resultant impetus ${ }^{2}$ is zero in the result of reciprocal "cancelling off" of oppositely directed impetuses. Another formulation of the definition of the distinguished observer (frame) remaining in the state of "absolute" rest and "perceiving" the global flow of time is such an observer (frame of reference) from which all distant galaxies located at the same distance drift away (become more distant) (as a result of the expansion of the universe) with the same speed (Smolin, 2014). This distinguished reference frame can be determined, at least in principle. Finally, the very evolution of the universe, its expansion from the moment of the Big Bang, fixes the cosmic, universal flow of time.

However, another method of defining the distinguished, "rest" reference frame in space exists. The reference point here is the cosmic microwave background radiation, a relic of the early stages of the universe's evolution (Weinberg, 1977; Davies, 1996; Barrow, 1997; Greene, 2005). This radiation coming from one side of the heavenly sphere is on average slightly "warmer", and has a lower wavelength (higher energy, violet shift) than

\footnotetext{
${ }^{1}$ Kinetic energy is expressed by the equation: $\mathrm{E}=\mathrm{mv}^{2} / 2$, where $\mathrm{E}$ is kinetic energy, $\mathrm{m}$ is the mass of the moving object and $\mathrm{v}$ is its velocity. ${ }^{2}$ Impetus is expressed by the equation: $\mathrm{p}=\mathrm{mv}$, where $\mathrm{p}$ is the vector of impetus (value and direction in three dimensions), $\mathrm{m}$ is mass and $\mathrm{v}$ is the vector of velocity.
} 
the radiation coming from the other side of the heavenly sphere (lower energy and temperature, red shift). On the basis of this fact, it has been calculated that the earth, together with the sun and the Milky Way, move with a speed equal to $0.5 \%$ of the speed of light in relation to the cosmic microwave background radiation, which constitutes our distinguished, "rest" reference frame. This frame is also distinguished in relation to the measurement of time flow. It is in relation to this fact that the universal, "absolute", global time of the universe is measured. It is this fact that determines the reference frame for the unconditional simultaneousness. All this seems to contradict the principle of relativity. However, global cosmic time independent of the reference frame that is universal for the entire universe appears in some cosmological models based on solutions of the equations of the general theory of relativity (for instance in the Robertson-Walker metrics).

In addition, the phenomenon of quantum entanglement and non-locality of quantum mechanics (Tegmark \& Wheeler, 2003; Penrose, 2002; Greene, 2005) seem to evidence non-local time. When a particle-antiparticle pair, for instance electron-positron pair (or a pair of photons), originates as a result of a quantum process, each of the elements of this pair won't have determined quantum properties, for instance spin orientation (or direction of polarization), according to the indeterminacy of quantum mechanics. A superposition of quantum states will take place, for instance: spin oriented in a given direction-spin oriented in the opposite direction (or two opposite polarizations). The only thing that is known about these particles/antiparticles is that these properties, for instance spin orientation, are opposite for both (anti)particles. This is called quantum entanglement. These (anti) particles can go away from each other in the opposite direction for a distance of, say, one million light years. Then one can determine at a chosen moment the spin orientation (or polarization) of one of the particles. Or one particle can simply interact with the surroundings (in my opinion, no conscious observer or special device is needed here). At the same moment, instantaneously, the spin (polarization) of the other particle will adopt the opposite value (see e.g., Penrose, 2002; Greene, 2005; Tegmark \& Wheeler, 2003). In other words, before an interaction with the surroundings of one of the particles in the pair, they do not possess determined quantum properties (spins, polarizations). Only the properties of the entire entangled system, particle-antiparticle, are determined. This phenomenon seems difficult to be reconciled with the relativity of time, which decidedly speaks for its global character and universality.

Nevertheless, the existence of the distinguished, "rest" reference frame (observer) in space, which experiences a global, universal for the whole universe flow of time, adds more support for the conception of a four-dimensional, congealed, timeless block of space-time.

\section{Thermodynamic Arrow of Time}

Thermodynamics explains where the arrow of time, that is, in other words, the immanent asymmetry of the time dimension in macroscopic systems composed of (very) many elements, comes from. It is inseparably associated with the increase in entropy (proportional to the number of microscopic states corresponding to the macroscopic state in which the system currently is; Prigogine, 1980; Prigogine \& Stengers, 1984; Penrose, 2002) and decrease of (thermodynamically defined) amount of information and degree of complexity of the system. However, this does not imply an existence of real flow of time, associated inseparably with the notion of the present, but only the fact that in the congealed block of space-time, entropy is lower at its one end, marked as "earlier", than at its opposite end, marked as "later". I ignore the fact that the separation (determination) of the macroscopic state, which is crucial in statistical thermodynamics to define entropy, amount of information and degree of complexity constitutes in its essence, to a large extent, subjective operation. Apart from this, the arrow of time related to the entropy increase only has a statistical character. The transition from a macroscopic state with lower entropy (containing less microscopic states) to a macroscopic state with higher entropy (containing more microscopic states) is simply more probable (although by a truly astronomic factor in complex systems) than the opposite transition. Nevertheless, the latter is in principle possible. In "systems" composed of just one element there would be no thermodynamic flow of time. Finally, thermodynamics contains no distinguished moment of the present. Summing up, the "thermodynamic universe" can also be presented in the form of a congealed, timeless block.

\section{4. "Freezing of Reality" as the Source of the Real Flow, Arrow of Time and the Present}

If time actually flows from the past to the future, then the present means the point on the time axis in which the 
physical world currently is. This is like with a tape recorder tape containing a record of music-we can univocally say at which point the tape has just been read out by the recorder, and thus which fragments of a piece of music have just been played. However, if we accept the determinism of the physical reality, then we can treat the four-dimensional space-time filling our universe together with matter and energy, when we look at it from the outside, as a congealed block that is not happening in time (as it contains time in itself), but simply is (lasts timelessly, whatever this might mean). In such a perspective, all time moments exist at once, "simultaneously", while the flow of time, past, present and future, are only an illusion of our mind. In other words, our tape recorder tape is not read out at all-both it itself and all the music recorded on it constitute a certain determined entity existing simultaneously in all its fragments from the beginning to the end, and it only seems to human consciousness that for not fully understood (but rather unimportant) reasons just that and not another fragment is read out, following the previous fragment and preceding the next fragment. It is astonishing how many physicists would sign willingly under such a vision of time.

However, this vision does not explain one simple, elementary thing: why (it seems to us) do we find ourselves (with the entire physical reality) just in this and not another point of time. In other words, this is the problem of anchoring in the present, between the past and the future in such a way that the point of anchoring moves from the past to the future. Therefore, the flow of time is reduced in this conceptualization to a shifting border (the present) between the past and the future.

Because no one actually knows what the flow of time consists in, I will present my own conception. I call it the "freezing of reality". As I mentioned before, the conception of the seen from the outside, congealed and stiff space-time is possible only under assumption of complete determinism of the laws of physics. However, if the contemporary (interpretation of) quantum mechanics is right, the world is ruled by indeterministic, probabilistic laws (Tegmark \& Wheeler, 2003; Hawking, 1988; Penrose, 2002; Greene, 2005; Chandrasekhar, 1998; Davies, 1996). Therefore, while the past is congealed, certain and unchangeable (these are most elementary attributes of the past), there exists in the future yet multiple chances of various events that can be specified only probabilistically. This, what happens in the present moment, is a transition from an uncertain, probabilistic future, to a certain, congealed past. The propagation of the wave-function (probability wave), determining for instance the probability of finding a particle (e.g., electron) associated with it in a given point of space, is strictly deterministic, while the equations describing it are symmetrical in time. However, the collapse and decoherence of the wave-func- tion are decidedly asymmetrical in regards to time. The collapse is a discrete, instantaneous "reduction" of the wave-function describing probabilistic distribution of different quantum states to one quantum state, for instance a concrete location of an elementary particle in space, as a result of a sudden interaction with the surroundings, for example a "measurement” (Penrose, 2002; Tegmark \& Wheeler, 2003; Greene, 2005). The decoherence is a gradual "reduction" of the wave-function, as a result of progressing interaction with the surroundings (Penrose, 2002; Tegmark \& Wheeler, 2003; Greene, 2005). In this process the number of possible quantum states continuously decreases in time, but is not reduced at once to one. For instance, the spherical probability wave associated with an electron emitted as a result of some process, after passing through a slit in a diaphragm located on the course of the propagation of the wave, becomes partly reduced. Namely, the number of places in space where the electron can be found becomes partly limited, although it is not yet strictly spatially localized. Apart from this, the wave can undergo instantaneous collapse on the diaphragm, which leads to complete localization of the electron, or only a partial reduction as a result of decoherence after passing through the slit in the diaphragm. Similarly, the probability wave of an electron bound in an atom, adopting the form of an orbital, becomes reduced in relation to the spherical probability wave freely propagating in space.

In my opinion, in principle neither the collapse nor decoherence requires "measurement" by a special device or the presence of a subjective observer (see below). This, what happens in the present, is a transition from an uncertain, probabilistic future to a certain, congealed past. And just this transition, occurring with the speed equal to the speed of the time flow (that is, speaking half-jokingly, one second per second), corresponds, in my opinion, to the movement of the point of the present along the time axis. To some extent, this resembles phase transition during the freezing of water. The liquid phase (water) characterized by chaotic movement of molecules that behave in a not fully predictable (at least in practice) way transits into the solid phase (ice) with molecules congealed in a determined place in the crystal network. In over-cooled water, this phase transition propagates in water starting from the centre of crystallization. Similarly, in my conception the present is the moving "wave front" associated with the "phase transition" of the uncertain future into the congealed past. The wave front of the phase transition can be located once only in one point on the axis of time (the present), univo- 
cally dividing it on the "solid" part (the past) and "liquid” part (the future). This "freezing of reality" in a natural way determines our "anchoring in reality" and the phenomenon of the real flow of time.

Of course, some wave-functions associated with elementary particles (or objects composed of them), for instance orbitals, did not undergo collapse/decoherence in the past, or their reduction as a result of decoherence was only partial. They "freeze" just in such, "not finished" form. Therefore, the completely frozen past contains "single" quantum states such as the determined spatial location or spin of an electron. In contrast, the partly frozen past contains wave-functions of different degrees of reduction/decoherence. Therefore, "frozen" does not mean "reduced to a single quantum state". The instantaneous or gradual reduction of the wave-function as a result of collapse or decoherence is irreversible, happening at the moment of the present. The "frozen" in the past, not reduced or only partly reduced wave-functions will never again undergo further reduction (collapse or decoherence) in this past. Of course, this reduction can occur, and frequently actually takes place, in the future (or basically in the present, "transforming" the future into the past). This process becomes consolidated ("recorded") in the "later" past. The past, once it has come into existence, remains unchanged. As no system in the universe is perfectly isolated, ${ }^{3}$ there always occurs even slight reduction of the wave-function. The stronger the interaction of a given particle (system) with the surroundings, the stronger the decoherence, and thus the more pronounced the arrow (irreversibility) of time. Therefore, the global time flow, occurring with an even pace, is not equivalent to the "local" arrow of time of an intensity proportional to the magnitude of the reduction of the wave-function in time. Incidentally, the global, cosmic flow of time is also independent of the thermodynamic arrow of time (see below), and also occurs in systems that are in the state of thermodynamic equilibrium.

Even a smallest quantum indeterminacy and its reduction in the result of collapse/decoherence of the wave-function can direct the evolution of the system towards a completely unpredictable course. This happens in particular in systems in which the so-called deterministic chaos prevails ("deterministic" means here "not taking into account the indeterminism of quantum mechanics”). In such systems (called "chaotic systems”), even a tiny (microscopic) change in initial conditions can have in not distant time huge, macroscopic effects (Gell-Mann, 1994; Coveney \& Highfield, 1991; Coveney \& Highfield, 1996; Stewart, 2002; Stewart \& Cohen, 1997). The earth's atmosphere constitutes a good example here. According to the commonly known "butterfly effect”, a movement of butterfly wings over Japan can elicit a hurricane in the United States.

It ought to be decidedly emphasized here that, in my opinion, the famous thought experiment known as the Schrödinger's cat paradox, requiring the presence of a conscious observer, is very misleading and simply wrong. In this experiment we enclose a cat in an isolated container together with a clump of radioactive substance and a detector of electrons connected to a phial filled with poisonous gas. The radioactive beta decay, associated with an emission of an electron, is in its essence, as a quantum phenomenon, purely probabilistic. This means that the fact of whether such a decay will take place within, say, a given concrete minute is exclusively a matter of chance. If an electron is emitted, it will be detected by the detector, which will lead to the breaking of the phial and the death of the cat. In the opposite case, the cat will remain alive. However, as long as we do not open the container, we will not be able to learn this. Therefore, the system will remain in superposition of two states: the decay took/did not take place, the electron was/was not detected by the detector, the poison was/was not released, the cat is dead/alive. In other words, the cat remains at the same time dead and alive. Only a conscious observer, after opening the container, will be able to learn which of these cases takes place, and thereby to lead to a collapse, reduction of the wave-function to a single state.

An involvement of a conscious observer here is completely unnecessary. The collapse/decoherence of the wave-function of an electron occurs at the moment of arriving of the probability wave associated with the electron to the detector. Precisely at this moment, precise determination of the electron location, release of the poisonous gas and death of the cat take place. If as a result of the collapse of the wave-function the electron becomes localized on the wall of the container, and not in the detector, or if after the electron emission it meets something on its way to the detector, the cat will remain alive. Therefore, there is no place here for any macroscopic quantum effects, nor is there a need for an external observer. The paradox disappears. The cat always remains univocally alive or dead. Macroscopic objects do not behave in the quantum manner. The freezing of reality always occurs basically on the quantum level.

Smolin (2014) and other authors postulated that a deeper theory comprising the entire universe, of which quantum mechanics is only a local approximation, will be strictly deterministic. Therefore, there will be no place

\footnotetext{
${ }^{3}$ Even an elementary particle in inter-galactic space interacts e.g., with the microwave background radiation and gravitational field of distant galaxies (Greene, 2005).
} 
in it for the collapse and decoherence of the wave-function. According to this conception, the indeterminacy of quantum mechanics in an arbitrarily separated experimental system would result from not taking into account the influence of the rest of the universe. Therefore, in this hypothetical theory, there would be no "freezing of reality", the present, arrow of time and real flow of time. However, as yet, this is pure speculation.

I mentioned above that it seems that there exists in the universe a certain distinguished, "rest" frame of reference, in relation to which the universal, cosmic time of the universe flows. Only in this reference frame does the present appear and the freezing of reality occur. It is not possible to present the universe with the "freezing of reality" in the form of a congealed block, as it is not known what will happen in the future (only the "frozen" past can be presented in the block form).

\section{Once Again about Thermodynamics and Entropy}

There remains a principal question: what is the relation between the direction of the above-defined "universal" flow of time and the thermodynamic arrow of time? I believe that the solution of this fascinating problem constitutes one of great challenges of modern physics. The fact that the universe "got going" from a state of extremely low entropy, and therefore this entropy perforce increased with the course of time, is crucial here; as, what might seem a paradox, the homogeneous (accidental, chaotic) distribution of matter in space is least probable in the gravitational field (Penrose, 2002; Greene, 2005; Barrow, 2006). Just such a distribution of elementary particles took place right after the Big Bang. During the evolution of the universe, matter exhibits a tendency to gather in aggregations on different levels of hierarchy: clusters of galaxies, galaxies, stars, planets, moons and asteroids. However, matter will end its existence in black holes, objects of maximal possible entropy (Penrose, 2002; Barrow, 2006). The entropy of a black hole is proportional to its size (strictly speaking: its surface) (Hawking, 1988; Penrose, 2002). It can increase further as a result of absorbing the surrounding matter (and energy) or the fusion of two black holes. Black holes can "evaporate" as a result of so-called Hawking radiation (Hawking, 1988; Baggott, 2014; Smolin, 2014). However, this process is enormously slow, and the larger the black hole, the slower the process is. On the other hand, the rate of black hole growth, if only as a result of absorption of matter or microwave background radiation filling the whole universe, is greater than the loss of its mass as a result of Hawking radiation. If the entropy of the universe adopted at the very beginning the maximal value (all matter and energy concentrated in one point, something like a black hole ${ }^{4}$ ), nothing would happen afterwards. However, we do not know whether the initial entropy results from necessity (laws of physics) or from accidental circumstances (chance). According to some theories, the overall energy of the universe is zero, as the positive energy associated with matter (its mass and kinetic energy) precisely counterbalances the negative energy of space resulting from the moving of objects endowed with mass in the gravitational field away from each other (Hawking, 1988, Smolin, 2014). It was suggested that the coming into being of the universe in fact consisted in the origination of information about the reciprocal distribution of matter and space (Korzeniewski, 2013b, 2015a, 2015b). A large amount of information means low entropy. Let us note that an apparently opposite situation takes place in systems, in which gravitation (or e.g., electrical force) between their elements is negligible. For instance, in a container with gas in it, the system has an immanent tendency to transition (associated with an entropy increase) from the uneven distribution of gas molecules (for instance, all molecules in the left half of the container) to an even distribution (gas molecules homogeneously, chaotically distributed over the whole container volume). However, in this case, practically no attractive force is present between gas molecules and the only interaction between them consists in unceasing collisions causing their rejection from each other or from the walls of the container. The evenly distributed elementary particles in the early universe formed a very unstable system because they mutually attracted each other via gravitation. Only the expansion of the universe prevented concentration of all matter in one point. With minimal disturbance, such particles (and later on atoms formed of them) started to form still greater and greater aggregations of matter, attracting by means of gravity still more and more new particles/atoms, resulting in the increased mass and gravitation of these aggregations. This was a self-driving process. These aggregations then began to fuse, forming still larger and larger aggregations, attracting through even greater gravity still more particles/atoms, resulting in the increase of their mass and gravitation. On the other hand, the kinetic energy of gas molecules in the container at room temperature is by several orders of magnitude greater than the energy associated with the tiny gravitational

\footnotetext{
${ }^{4}$ The initial singularity within the general theory of relativity is in its essence an opposition of a black hole (in the initial singularity, the Weyl tensor equals zero, while in a black hole it tends towards infinity) (Penrose, 2002).
} 
attraction between them. Therefore, in this system the even distribution of molecules (representing the state of thermodynamic equilibrium of maximum entropy) is stable.

Modern physics also offers answers to the question of where the initial even (chaotic) distribution of matter in the universe came from. Straight after the Big Bang, the universe was so small, dense and hot that the kinetic energy of elementary particles was so big that it exceeded the energy (strength) of gravitational, electrical and even strong nuclear forces. Therefore, similarly to gas molecules in a container, they were distributed more or less evenly in space. As a result of the enormously huge and sudden expansion of (the space of) the universe, in a process called inflation (Hawking, 1988; Barrow, 1997; Greene, 2005), it significantly cooled down. This led to the kinetic energy of elementary particles falling dramatically and particular forces arose. Quarks combined to form protons and neutrons. Electrons recombined with hydrogen and helium nuclei, which became electrically neutral atoms. As a result of this event, the universe becomes transparent and microwave background radiation was released (Weinberg, 1977; Barrow, 1997; Greene, 2005; Smolin, 2014). In macroscopic scales of distance, only gravitation remained on the scene, leading to the formation of matter aggregations on different scales of magnitude, as discussed above.

It is worth adding that life does not exist in systems with minimal entropy, as one might suppose. Life exists in systems with intermediate entropy between the minimum entropy of evenly distributed elementary particles in the gravitational field after the Big Bang and the maximum entropy of black holes, in systems where the intensive production of entropy (dissipation of energy) takes place (Schrödinger, 2002; Prigogine \& Stengers, 1984). Therefore, living organisms are an example of complex, hierarchically organized dissipative structures, ordered in space and time (Prigogine, 1980; Prigogine \& Stengers, 1984) that are found also in inanimate nature (e.g., convective currents in liquids).

\section{The Phenomenon of Life and Arrow of Time}

Coming back to the main topic of this article- the problem of the real flow of time, arrow of time and the present- they have basic significance for the phenomenon of life as well as psyche and self-consciousness. Generally, complexity appears as a result of self-organization occurring according to the direction of the flow of time (Coveney \& Highfield, 1996; Stewart \& Cohen, 1997). This does not contradict the second law of thermodynamics because the entropy of a complex system diminishes locally at the cost of an increase in the entropy of the surroundings. In the case of life, current functioning of a living individual, its development from the stage of a fertilized egg cell (zygote) to the adult stage (ontogenesis), its reproduction and biological evolution (phylogenesis), we deal with a clear arrow of time and irreversibility of processes. Both ontogenesis and phylogenesis are enormously complex processes decidedly proceeding only "from the past to the future". In mutation generation, which is crucial for biological evolution, a key role is played by probabilistic and irreversible quantum processes (collapse, decoherence of the wave-function) occurring on the level of quantum chemistry. Biological systems, living individuals in particular, can be distinguished from inanimate systems (and also from their parts, for instance organs and cells, as well as from complex devices built by humans) in the frame of the cybernetic paradigm, in which a key role is played by negative and positive feedback (Korzeniewski, 2001, 2005, 2013a). Mutually interconnected with each other negative feedbacks, which maintain the homeostasis of a living individual, control its functioning and decide about its identity, "happen" in time. Similarly, the reproduction, identical with the superior positive feedback "sustained" by the network of negative feedbacks, constituting the superior aim of (the identity of) every individual and an indispensable element of biological evolution, is irreversible in time, while the fitness of a living individual (another key element of evolution through natural selection) is continuously tested in every concrete moment of time (the present).

\section{Self-Consciousness and Arrow of Time}

Regarding the psychic individual, endowed with self-consciousness, of great importance is both subjective perception of the flow of time and its irreversibility (arrow of time), and the "anchoring" of the mind in the current moment of time, the present. Human psyche evolves during the entire life of a human being. Its content can also change, frequently drastically and sharply, "from a while to a while", during everyday life. This is associated with the phenomenon of attention that directs the "light of consciousness" on different objects, both external and internal. The acquiring of memory and experience is an irreversible process. We remember the past, but not the future. We immediately experience the present. The biochemical and biophysical processes in neurons that underlie subjective psyche and self-consciousness, for example the transport of sodium and potassium ions across 
the cellular membrane of an axon while conducting neural impulses, occur to a large extent on the quantum level. Therefore, the quantum "freezing of reality" also directly applies to them. In addition, a self-mapping, directing on itself, forming its own representation within itself by the "cognitive" centre in human brain that was proposed as the neural mechanism underlying the phenomenon of self-consciousness (subjective psyche) (Korzeniewski, 2010, 2013a, 2015a, 2015b) requires the flow of time and its irreversibility. This conception also assumes a notion of the psychological present, maybe of a finite, non-zero duration, for example, the 0.5 seconds (Davies 1996), needed to close the loop of receiving signals by the cognitive centre from itself by itself (Korzeniewski, 2010, 2013a, 2015a, 2015b).

It has been argued that the dynamic complexity of matter in space and time underlying the phenomenon of life (biological individual) and self-consciousness (psychical individual) exists in an equally objective and real way as matter, space and time themselves (Korzeniewski, 2015b). The flow of time, its irreversibility (arrow of time), and the present should be regarded as equally real and objective. These phenomena are present both on a purely physical level and in our subjective feeling.

\section{Summary}

The mathematical formalism of great dynamic theories in physics, among the Newton's mechanics, Maxwell's electromagnetism, Einstein's special and general relativity as well as quantum mechanics, is symmetrical regarding time. Therefore, in agreement with these theories, the four-dimensional space-time of the universe can be in principle presented as a congealed timeless block, in which time is only one dimension, and in which there is no place for the unidirectional and irreversible flow of time (arrow of time) and the present. The arrow of time related to entropy increase is present in thermodynamics, a branch of physics that deals with systems composed of many elements. However, also in this case, time can be presented as a timeless, congealed block, on the one end of which (called "the past") entropy is smaller, while on the other end (in the future) it is greater. On the other hand, we sense a clear psychological arrow of time, directed from the past to the future, as well the current moment (the present) that separates them. This article postulates that these phenomena exist in a completely real and objective way (as real and objective as space and matter). The irreversible process, called the "freezing of reality", directly associated with the collapse or decoherence of the wave-function as a result of its interaction with the surroundings, is responsible for this. It needs neither any measuring apparatus nor a subjective, conscious observer to occur. The universe with the "freezing of reality" cannot be described as a congealed, fourdimensional, timeless block—such a block can represent only the "frozen" past, but not the unpredictable future. The present determines the border between them moving together with the flow of time along the time axis, which remains in agreement with the global irreversible time universal for the entire universe. The proposed "quantum arrow of time" constitutes the basis of the thermodynamic arrow of time (evolution of the distribution of matter in the universe from the even distribution just after the Big Bang, through galaxies, stars and planets, to still greater and greater black holes), the biological arrow of time (current functioning of living individuals, individual development—ontogenesis, reproduction and biological evolution—phylogenesis), as well as the psychological arrow of time (subjective feeling of the unidirectional and irreversible flow of time, of the present as well as the phenomenon of self-consciousness).

\section{Acknowledgements}

Faculty of Biochemistry, Biophysics and Biotechnology, Jagiellonian University is a beneficiary of KNOW program.

\section{References}

Baggott, J. (2014). Farewell to Reality: How Modern Physics Has Betrayed the Search for Scientific Truth. Pegasus.

Barrow, J. D. (1997). The Origin of the Universe. New York: Basic Books.

Barrow, J. D. (2006). The Infinite Book. The Short Guide to the Boundless, Timeless and Endless. New York: Vintage.

Chandrasekhar, B. S. (1998). Why Things Are the Way They Are. Cambridge: Cambridge University Press.

Coveney, P., \& Highfield, R. (1991). The Arrow of Time. The Quest to Solve Science's Greatest Mystery. London: Flamingo.

Coveney, P., \& Highfield, R. (1996). Frontiers of Complexity. The Search for Order in a Chaotic World. New York: Ballantine Books. 
Davies, P. (1996). About Time: Einstein’s Unfinished Revolution. New York: Simon \& Schuster.

Gell-Mann, M. (1994). The Quark and the Jaguar. Adventures in the Simple and the Complex. New York: W.H. Freeman.

Greene, B. (2005). The Fabric of the Cosmos. Space. Time. And the Texture of Reality. New York: Vintage.

Hawking, S. W. (1988). A Brief History of Time: From the Big Bang to Black Holes. New York: Bantham Books.

Korzeniewski, B. (2001). Cybernetic Formulation of the Definition of Life. Journal of Theoretical Biology, 209, $275-286$. http://dx.doi.org/10.1006/jtbi.2001.2262

Korzeniewski, B. (2005). Confrontation of the Cybernetic Definition of a Living Individual with the Real World. Acta Biotheoretica, 53, 1-28. http://dx.doi.org/10.1007/s10441-005-7000-7

Korzeniewski, B. (2010). From Neurons to Self-consciousness. Amherst, MA: Prometheus Books.

Korzeniewski, B. (2013a). Formal Similarities between Cybernetic Definition of Life and Cybernetic Model of Self-Consciousness: Universal Definition/Model of Individual. Open Journal of Philosophy, 3, 314-328.

http://dx.doi.org/10.4236/ojpp.2013.32049

Korzeniewski, B. (2013b). Magic of Language. Open Journal of Philosophy, 3, 455-465. http://dx.doi.org/10.4236/ojpp.2013.34067

Korzeniewski, B. (2015a). Field of Meaning. Saarbrücken: Lambert Publishing Company.

Korzeniewski, B. (2015b). Mind-Body Problem: Does Complexity Exist Objectively? Open Journal of Philosophy, 5, 351364. http://dx.doi.org/10.4236/ojpp.2015.56043

Penrose, R. (2002). The Emperor's New Mind: Concerning Computers, Minds, and the Laws of Physics. Oxford: Oxford University Press.

Prigogine, I., \& Stengers, I. (1984). Order out of Chaos. London: Heinemann.

Prigogine, I. (1980). From Being to Becoming. San Francisco, CA: W. H. Freeman.

Schrödinger, E. (1992). What Is Life? Cambridge: Press Syndicate of the University of Cambridge. http://dx.doi.org/10.1017/CBO9781139644129

Smolin, L. (1997). The Life of the Cosmos. Oxford: Oxford University Press.

Smolin, L. (2014). Time Reborn: From the Crisis in Physics to the Future of the Universe. London: Mariner Books.

Stewart, I., \& Cohen, J. (1997). Figments of Reality. The Evolution of the Curious Mind. Cambridge: Cambridge University Press. http://dx.doi.org/10.1017/CBO9780511541384

Stewart, I. (2002). Does God Play Dice? The New Mathematics of Chaos. Hoboken, NJ: Wiley-Blackwell.

Tegmark, M., \& Wheeler, J. A. (2003). 100 Years of the Quantum Mysteries. Scientific American, 284, 68-75. http://dx.doi.org/10.1038/scientificamerican0201-68

Weinberg, S. (1977). The First Three Minutes. New York: Basic books.

\section{Scientific Research Publishing}

\section{Submit or recommend next manuscript to SCIRP and we will provide best service for you:}

Accepting pre-submission inquiries through Email, Facebook, LinkedIn, Twitter, etc.

A wide selection of journals (inclusive of 9 subjects, more than 200 journals)

Providing 24-hour high-quality service

User-friendly online submission system

Fair and swift peer-review system

Efficient typesetting and proofreading procedure

Display of the result of downloads and visits, as well as the number of cited articles

Maximum dissemination of your research work

Submit your manuscript at: http://papersubmission.scirp.org/ 